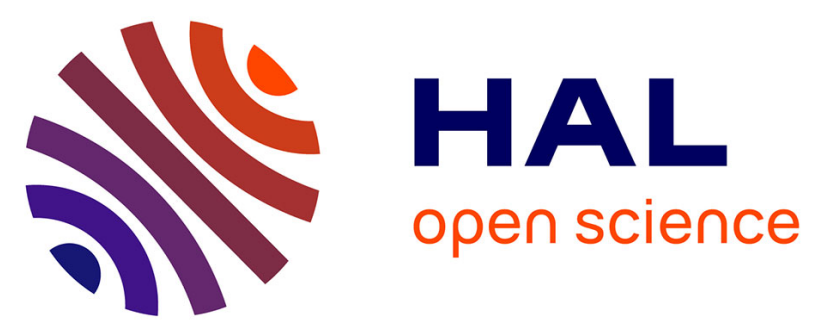

\title{
Light induced charge and energy transport in nucleic acids and proteins: general discussion
}

Amitabha Chattopadhyay, Richard Cogdell, Carlos Crespo-Hernández, Ankona Datta, Arijit De, Stefan Haacke, Mahesh Hariharan, John Helliwell, Ashley Hughes, Roberto Improta, et al.

\section{To cite this version:}

Amitabha Chattopadhyay, Richard Cogdell, Carlos Crespo-Hernández, Ankona Datta, Arijit De, et al.. Light induced charge and energy transport in nucleic acids and proteins: general discussion. Faraday Discussions, 2018, 207 (9), pp.153 - 180. 10.1039/C8FD90004C . cea-01881626

\section{HAL Id: cea-01881626 https://hal-cea.archives-ouvertes.fr/cea-01881626}

Submitted on 23 Oct 2019

HAL is a multi-disciplinary open access archive for the deposit and dissemination of scientific research documents, whether they are published or not. The documents may come from teaching and research institutions in France or abroad, or from public or private research centers.
L'archive ouverte pluridisciplinaire HAL, est destinée au dépôt et à la diffusion de documents scientifiques de niveau recherche, publiés ou non, émanant des établissements d'enseignement et de recherche français ou étrangers, des laboratoires publics ou privés. 


\title{
Light induced charge and energy transport in nucleic acids and proteins: general discussion
}

\author{
Amitabha Chattopadhyay, (iD) Richard Cogdell, (D) \\ Carlos E. Crespo-Hernández, (iD Ankona Datta, Arijit De, (iD \\ Stefan Haacke, (D) Mahesh Hariharan, John Helliwell, (D) Ashley Hughes, \\ Roberto Improta, Mike Jones, Joshy Joseph, Tolga Karsili, Bern Kohler, \\ Retheesh Krishnan, Anvy Kuriakose, Mahil L, Dimitra Markovitsi, (D) \\ Himani Medhi, Ganga Periyasamy, (D) P. I. Pradeepkumar, \\ Priyadarshi Roy Chowdhury, Manas Sarangi, Igor Schapiro, (iD \\ Gebhard F. X. Schertler, Ilme Schlichting, Javier Segarra-Martí, (D) \\ Rajaram Swaminathan, (D) Vishnu V, Rienk van Grondelle, \\ Ravi Kumar Venkatraman, Ravindra Venkatramani and Anthony Watts
}

\section{DOI: $10.1039 / C 8 F D 90004 C$}

Amitabha Chattopadhyay opened the discussion of the paper by Gebhard F. X. Schertler: Do you have an idea of the timescale of the dynamics of these water molecules in GPCR structures?

Gebhard F. X. Schertler replied: This depends on the water molecules that you are referring to. The water molecules close to the retinal will respond very fast. From looking at the time resolved measurements with bacteriorhodopsin, we know that it is faster than 16 nanoseconds. I expect it to be on the picosecond timescale. Water molecules in the hydrogen bond network connecting to the $\mathrm{G}$ protein binding site will rearrange in the same time frame as the conformational changes. In rhodopsin this ranges from microseconds to milliseconds.

Richard Cogdell remarked: I agree that water molecules are important. Why do you think you would use water in this space rather than a larger amino acid? Water gives you a much greater structural flexibility and a faster timescale. Do you think that is a reasonable way to think about it?

Gebhard F. X. Schertler responded: As I pointed out, water molecules can have many roles inside a protein. I focused on the highly occupied water sites. This gives little information about the dynamics and exchange rates of the water molecules. It also does not mean that less ordered water molecules inside the protein are not important and so they could exist if the space allows it. I think you are implying 
that water molecules are important for the transition between conformational states and their dynamics, which I think is a reasonable assumption.

Ankona Datta asked: You mentioned that the water molecules in the ligand binding sites provide some stiffness; could you elaborate on that? Do you mean that water is providing structure to the active sites?

Gebhard F. X. Schertler answered: In the figures that I have included in the manuscript it is clear that some water molecules in the ligand binding site exhibit a structural role, but I am also convinced that some can play a role in the chemical reaction mechanism in the active site. There are programs that are used today in structure based drug discovery to evaluate and predict the role of water molecules in ligand binding sites. ${ }^{1}$

1 A. Bortolato, B. G. Tehan, R. T. Smith and J. S. Mason, Methods Mol. Biol., 2018, 1705, 207232.

Rienk van Grondelle queried: I was wondering about the role of the water molecules in these systems. For instance, is there any evidence of charge transfer in the excited system?

Gebhard F. X. Schertler responded: At this moment all of our measurements are long after the charge separation, which happens upon the photo-excitation that puts the molecule in the exited state molecule landscape. In order to observe this, free electron laser measurements would be ideal, but we have not yet carried out these ultrafast measurements. Theory and spectroscopy puts these events between 100 and 500 fs.

Stefan Haacke commented: There has been a lot of work on dehydrated membranes but at the time it was not known which water molecules were removed by dehydration. Can you speculate what dehydration does to the molecules?

Gebhard F. X. Schertler replied: Dehydration clearly influences the ability of the protein to reach its full active conformation. This has been shown by neutron scattering and other methods for retinal proteins and a number of other membrane proteins. The exact mechanism of this observation is not completely understood. In my opinion even the removal of a sufficient number of exchangeable surface water molecules could be enough for the stabilisation of the active conformation to be inhibited. In my opinion, in my work it is less likely that the internal water molecules were affected as they would have required more severe dehydration which would have likely led to protein denaturation, which was not observed in these experiments.

Dimitra Markovitsi enquired: How do you expect the behaviour of the water molecules to change as you go from an ordered crystal to a macroscopically less ordered system, as may be the case for a biological system?

Gebhard F. X. Schertler answered: The outer hydration shell of the protein will increase a little bit, however one must consider that the protein contacts in 
a crystal only affect a small part of the surface. I expect the internal water molecule dynamics to be very similar.

Anthony Watts remarked: GPCRs are very mobile and going from $\mathrm{R}$ to $\mathrm{R}^{*}$ traverses many energy minima. Are you suggesting that there are different water networks for each minimum? You have said on page 5 of your paper that this implies just two types of networks.

Gebhard F. X. Schertler replied: This is a very important question. I am implying that the clearly distinct water mediated hydrogen bond networks in the ground state and the fully activated meta II state make an important contribution to the minimal energy of these states in the energy landscape of the reaction. It is also likely that other intermediate states see important contributions to their minimal states from similar hydrogen-bond networks. It is important to mention here that not only our observed water molecules but also less ordered, exchangeable water molecules could contribute to the stabilization of an intermediate. In addition, we believe that the exchangeability of water molecules in the hydrogen-bond networks is important for the rates of the transitions between states and is therefore important for shaping the energy landscape of GPCRs.

Mike Jones asked: How tightly locked in are these water molecules? Can you exchange them with $\mathrm{D}_{2} \mathrm{O}$ and do you see any effects from that?

Gebhard F. X. Schertler responded: Many water molecules inside a protein are exchangeable, with different rates observed. However, I have not done any experiments using $\mathrm{D}_{2} \mathrm{O}$.

John Helliwell communicated: With reference to the sentence beginning "After photo-activation, we can..." in the sub-section "Water molecules are key..."; a quantification of the atoms' positional errors is needed in order to statistically judge their dynamical movement. A web tool for such a calculation based on the Cruickshank diffraction precision index (dpi), i.e. when full matrix inversion isn't viable due to the atomic resolution diffraction data not being measurable, is available at http://cluster.physics.iisc.ernet.in/dpi/. ${ }^{1}$

1 K. S. D. Kumar, M. Gurusaran, S. N. Satheesh, P. Radha, S. Pavithra, K. P. S. Thulaa Tharshan, J. R. Helliwell and K. Sekar, J. Appl. Crystallogr., 2015, 48, 939-942.

Gebhard F. X. Schertler communicated in reply: This is a very good suggestion.

John Helliwell communicated: In the conclusions in your paper you have mentioned that you will be able to analyse key water interactions in "much more detail". Since the diffraction resolution of the crystals is intrinsically at a limit does "much more detail" refer to time-resolution? What time-resolution improvement is still needed for this important molecular system?

Gebhard F. X. Schertler communicated in response: What I mean is mainly time resolution, as in the bacteriorhodopsin case we have already reached $1.5 \AA$ in spatial resolution. The next step is to carry out experiments with the FEL in the picosecond 
and subpicosecond time range. This will first reveal the role of the water molecules in the very first intermediate after isomerization. The sub-femtosecond measurements can even access the exited state of the protein and the transition state and formation of the first intermediate. I speculate that in all of these states we will see changes in the closest water molecules. I see them as part of the active site.

Priyadarshi Roy Chowdhury communicated: What are the effects of varying the timescale on the dynamics of water molecules in the photo transduction of retinal proteins?

Gebhard F. X. Schertler communicated in reply: As pointed out in the paper, there are different classes of water molecules. Some are in the orthosteric binding site of ligands, influencing the mobility and selectivity of the binding pocket. The second class of waters can be directly involved in enzymatic mechanisms, as pointed out in the paper. Both classes will respond very fast to photo-activation changes. The third class of water molecules between the allosteric binding site and the ligand binding site are important for the conformational change and for shaping the energy landscape that defines the change between inactive and active states. These water molecules will respond more slowly, often within microseconds to milliseconds, coinciding with the conformational change dynamics.

Priyadarshi Roy Chowdhury further communicated: In the case of a biological system, what is the most convenient method to study the fluctuation behaviour of water molecules?

Gebhard F. X. Schertler communicated in response: Time-resolved difference Fourier transform infrared methods have been widely used. The broadness of the infrared peaks is a serious limitation. New possibilities carrying out fast FEL measurements at room temperature are an amazing new tool that enable us to learn more about the dynamics, at least of the highly ordered water molecules in a structure.

Tolga Karsili opened the discussion of the paper by Arijit De: For Venus, the returned electronic absorption and emission spectra exhibit a more intense emission peak in comparison to the absorption peak, which is in obvious contrast to what is observed for wild type-GFP. The authors attribute this observation to a more stable anionic form of the chromophore ( $c f$. the protonated form) which results in a greater initial Boltzmann population in the anionic form in the electronic ground state. Although plausible, the differing local protein environment in wild type-GFP $v s$. Venus may lead to modifications in the energetics of the bright and dark vertically excited states of the chromophore, thereby modulating the intensities observed in the absorption spectrum. Are the authors able to rule this out?

Arijit De replied: We have not ruled this possibility out. The schematic of the PES that we qualitatively proposed (Fig. 11) describes only one of the three possibilities (see Section 3.3.4), which is based on inverting the relative ground state Boltzmann population in the two forms (protonated form and neutral form) of the chromophore in the two proteins (Venus vs. wt-GFP) due to perturbation by local environment. It is certainly possible that the same local environment tunes the 
Frank-Condon factor of the chromophores in these two proteins, leading to very different absorption intensities. In addition, there could also exist very different rates of non-radiative relaxation leading to differences in fluorescence intensities; we have suggested this as an alternate mechanism (see the paragraph beginning "(2) There is a second..." in Section 3.3.4).

Tolga Karsili asked: The authors propose a slower rate for ESIPT in the chromophore of Venus in comparison to that in wild type-GFP. Are the authors able to quantity this by a kinetic isotope effect? Such a study may also reveal if the rate is limited by a simple thermal motion over the barrier or H-atom tunneling through the barrier.

Arijit De answered: Yes, that is a good suggestion, thank you. In fact, the very first work on ESPT in wt-GFP carried out by Boxer et al. ${ }^{1}$ discussed similar isotope effect experiments.

1 M. Chattoraj, B. A. King, G. U. Bublitz and S. G. Boxer, Proc. Natl. Acad. Sci. U. S. A., 1996, 93, 8362-8367.

Bern Kohler remarked: I would like to hear more about your reasons for ruling out Raman scattering from the solvent (water) as the source of the weak peak at $462 \mathrm{~nm}$ seen in the emission spectrum. A peak at $462 \mathrm{~nm}$ is labeled in the emission spectrum recorded at an excitation wavelength of $400 \mathrm{~nm}$ in the middle panel of Fig. 6, but although the text states that this feature is also seen when exciting at $374 \mathrm{~nm}$, the only peak in the left panel of Fig. 6 between 400 and $500 \mathrm{~nm}$ is clearly shifted to a shorter wavelength, and thus appears to maintain the same constant frequency offset of approximately $3400 \mathrm{~cm}^{-1}$ relative to the excitation wavelength expected for Raman scattering by water. Raman peaks are, of course, difficult to detect in the presence of strong emission, but the weak absorbance of your sample at 374 and $400 \mathrm{~nm}$ coupled with the visible noise in the emission spectra in Fig. 6, and especially in Fig. 9, suggest that the emission is sufficiently weak for the water Raman band to be detectable. You write that you rule out Raman scattering based on the observation of a nanosecond lifetime, but such a response could arise from fluorescent impurities and could be present alongside the prompt scattering that originates from the Raman signal.

Arijit De replied: It is not the case that the Raman contribution is ruled out but, in addition to Raman scattering, there is also a (weak) fluorescence contribution leading to a longer lifetime component, as Raman scattering would yield a very short lifetime which is on the order of the instrument response, ${ }^{1}$ as we discussed in our paper. We have further pursued this issue recently. Fig. 1 below shows the fluorescence/scattering spectra of Venus (left panel) and pure buffer (middle panel); in the third panel we show the fluorescence spectrum after subtraction of the scattering spectrum (followed by normalizing the intensity). It is clear that the Raman peak shifts with excitation wavelength, which is contributed to by water $\left(\sim 3400 \mathrm{~cm}^{-1}\right)$. However, in addition to the Raman peak, there is broad fluorescence in this wavelength region. The fluorescence (462 nm) excitation spectrum (Fig. 9, left panel), as well as the UV-Vis absorption spectrum (Fig. 8, right panel), confirms that there exists an excitation/emission pair (400 nm/462 nm), as we discussed in our 

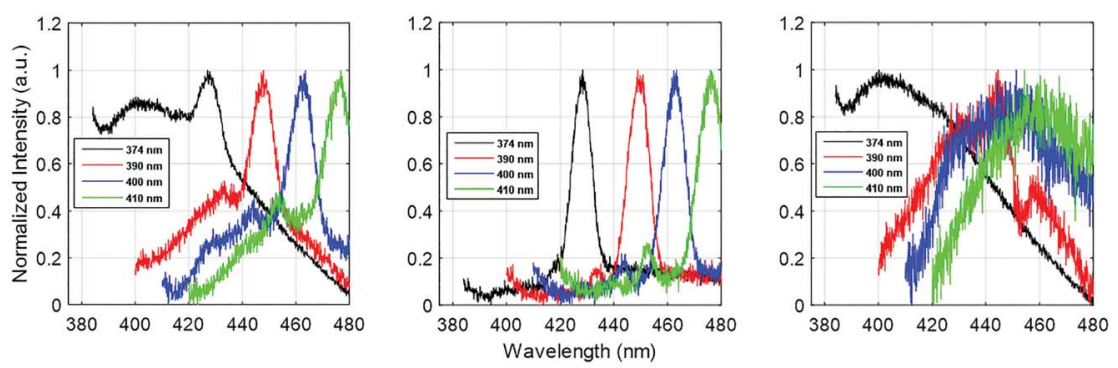

Fig. 1 Fluorescence/scattering spectra of Venus (left panel) and pure buffer (middle panel). Right panel: Fluorescence spectrum after subtraction of the scattering spectrum (followed by normalizing the intensity).

paper. Now, in order to estimate the fluorescence lifetime (at $462 \mathrm{~nm}$ ), the correct approach would have been to excite the protein with a $\sim 400 \mathrm{~nm}$ laser diode instead of a $374 \mathrm{~nm}$ laser diode. Quite interestingly, the $9.74 \mathrm{~ns}$ component is most likely due to emission from tryptophan ${ }^{2}$ which absorbs over a broad region with a peak at $\sim 280 \mathrm{~nm}$ (the $374 \mathrm{~nm}$ laser diode excites the red-edge of the tryptophan absorption). In addition, as shown in the right panel of the attached figure, there is also an emission at $\sim 400 \mathrm{~nm}$ (apart from the absorption at $\sim 400 \mathrm{~nm}$ ), which most likely arises from a broad absorption with a peak at $\sim 350 \mathrm{~nm}$ (inset in Fig. 7, left panel). These findings further complicate the assignment of the electronic states, which is what we are presently working on.

1 Y. Silori, S. Dey and A. K. De, Chem. Phys. Lett., 2018, 693, 222-226.

2 E. Gudgin, R. Lopez-Delgado and W. R. Ware, Can. J. Chem., 1981, 59, 1037-1044.

Roberto Improta asked: Can you please give some additional explanation about the origin of protein-backbone fluorescence? Is it necessarily associated with CT states?

Arijit De $\dagger$ answered: The origin of the protein-backbone fluorescence is believed to be due to the excitation of a lone pair of electrons on the carbonyl oxygen of the $\mathrm{C}=\mathrm{O}$ group that is present in the peptide bond, according to Guptasarma and coworkers and numerous other groups (e.g. Kaminski and coworkers, Dobson and coworkers). It is thought that this lone pair can jump across to other hydrogen bonded peptide bonds (in protein secondary structures), leading to their substantial delocalization and a consequent long-wavelength excitation (peaking at $\sim 387 \mathrm{~nm}$ but extending up to well past $400 \mathrm{~nm}$ ) and long wavelength emission (peaking, sometimes with multiple peaks, in a protein secondary structure content-dependent fashion between 425 and $550 \mathrm{~nm}$ ). Recently, some evidence was reported by the groups of Muschol and Uversky and colleagues in 2017 , which suggested that some amino acids can also exhibit this fluorescence. It is considered likely that this lone pair can also exhibit fluorescence through hydrogen bonding to water (i.e. the solvent surrounding proteins), albeit with a much poorer intensity than what is seen with proteins. Thus, some (though not all, e.g. not proline) amino acids display this fluorescence, although they possess no peptide bonds, because of the behavior of the carbonyl oxygen 
and the presumed exchange of the electrons in the lone pair with the water molecules with which they are hydrogen-bonded. The extinction coefficients and quantum yields, however, are very low. There is a possibility that the mechanism also involves some form of excited state proton transfer, as demonstrated by Kaminksi and colleagues in 2015. Furthermore, Venkatramani has suggested in a theoretical treatment that such a low intensity fluorescence could also involve charge transfer (DOI: 10.1039/c7fd00203c). However, neither work has excluded the possibility of the charge transfer (whether with or without associated proton transfer) involving the lone pair of electrons on the carbonyl oxygen of the peptide bond, as originally suggested by Guptasarma and coworkers. ${ }^{1,2}$

1 A. Shukla, S. Mukherjee, S. Sharma, V. Agrawal, K. V. Radha Kishan and P. Guptasarma, Arch. Biochem. Biophys., 2004, 428, 144-153.

2 P. Guptasarma, Arch. Biochem. Biophys., 2008, 478(2), 127-129.

Anthony Watts queried: Are you sure that your protein is pure? The small impurities shown in Fig. 2 and 3 may be the origin of the low intensity fluorescence.

Arijit De $\dagger$ responded: The two minor impurities seen in Fig. 2 are known contaminants of Imidazole Metal Affinity Chromatographic (IMAC) protein purification; the contaminant of $\sim 66 \mathrm{kDa}$ size is the protein known as ARNA and the $\sim 35 \mathrm{kDa}$ contaminant is the protein known as SlyD. Both proteins are typically present at less than 2 percent of the total population, and often at less than 1 percent. They are both standard proteins with no known special spectroscopic characteristics other than those that are seen in all proteins. In Fig. 2, the minor contaminants consist of higher molecular weight (e.g. aggregates) as well as lower molecular weight (e.g. degraded) species of a hydrodynamic volume, which differs to that of the major species (Venus) undergoing purification. Typically, contaminants showing up in SDS-PAGE are removed upon gel filtration chromatography because the probability of two proteins of different polypeptide mass having the same hydrodynamic volume is negligible, except where one (or both) of them happens to be multimeric, with coincident multimeric molecular weights. In this case, the Venus protein is monomeric and the other two proteins are also monomers. Therefore, as gel filtration chromatography was performed after SDSPAGE electrophoretic evaluation of Venus, there is no chance of either of the two contaminating proteins observed during electrophoresis also happening to contaminate Venus by co-eluting with Venus during chromatography. As the spectroscopy experiments were performed after chromatographic purification which would remove these contaminants (and also because the contaminants themselves have no scope for displaying a Venus-like fluorescence) there is no likelihood whatsoever of them being responsible for the low intensity fluorescence. However, one possible additional fact needs to be considered, and discarded (if necessary), before this question is fully answered, and this relates to the possibility of blue (peptide bond-derived) fluorescence. All proteins exhibit this fluorescence, with different quantum yields, and so if Venus displays this

$\dagger$ This response was prepared in collaboration with one of the authors of the paper, Purnananda Guptasarma, Department of Biological Sciences, Indian Institute of Science Education and Research (IISER) Mohali. 
fluorescence, so would every other protein, irrespective of whether or not it contains any aromatic or other (e.g. GFP-like) chromophore/fluorophore. From this viewpoint, any/all contaminants would exhibit low intensity fluorescence in proportion to their presence and quantum yields. Once again, as the contaminants are present in low amounts, any contribution to blue fluorescence would be correspondingly low and, therefore, the low intensity fluorescence would still be largely derived from Venus, even if it was due to blue fluorescence from peptide bonds, because Venus is the major component of the population.

Retheesh Krishnan remarked: It seems that for Venus the $462 \mathrm{~nm}$ peak exhibits a lifetime of $9.74 \mathrm{~ns}$. This lifetime seems to be too high for the argument you put forward, namely ESPT. However a further examination of Table 3 shows that the average reported lifetime is $1.48 \mathrm{~ns}$. Can you please comment on this?

Arijit De replied: This 9.74 ns component is most likely due to emission from tryptophan ${ }^{1}$ which is present at the $57^{\text {th }}$ position in the amino acid sequence (see Section 2.1 in the paper).

1 E. Gudgin, R. Lopez-Delgado and W. R. Ware, Can. J. Chem., 1981, 59, 1037-1044.

Ravi Kumar Venkatraman noted: It is quite interesting that the protein's environment can selectively stabilize the protonated or deprotonated form of the chromophore embedded in it. As you propose that the relative intensities of dual fluorescence arise from the Boltzmann distribution of populations, would you expect that a temperature dependent experiment would reveal the mechanism?

Arijit De answered: I don't think that a temperature dependent study would reveal this as the required elevated temperature would be so high that the protein would unfold (or even degrade).

Arijit De opened the discussion of the paper by Stefan Haacke: (1) In 1991, Shank, Mathies and co-workers ${ }^{1}$ showed (using pump-probe spectroscopy) that the initial isomerization (from rhodopsin to photorhodopsin) was complete after $200 \mathrm{fs}$. Later on, in 2005 Mathies and co-workers ${ }^{2}$ showed (using FSRS) that initial $200 \mathrm{fs}$ isomerization proceeds via a conical intersection and that the C-H HOOP (and not the $\mathrm{C}=\mathrm{C}$ torsion) drives this rapid internal conversion. Subsequently, in 2010 Cerullo, Mathies and co-workers ${ }^{3}$ demonstrated (using pump-probe spectroscopy) that the fast internal conversion takes place via the $\mathrm{C}=\mathrm{C}$ torsion (and not the $\mathrm{C}-\mathrm{H}$ HOOP), which is contradictory to the HOOP driven model. Is there any general consensus on how to identify the reaction coordinate $(\mathrm{C}-\mathrm{H}$ HOOP $v s . \mathrm{C}=\mathrm{C}$ torsion$)$ ?

(2) If you were to carry out a time-frequency analysis of this oscillatory data (along the waiting/population time), do you think you would see high frequency oscillations evolving into low frequency ones, thus revealing mode-mode coupling?

1 R. W. Schoenlein, L. A. Peteanu, R. A. Mathies and C. V. Shank, Science, 1991, 254, 412-415. 2 P. Kukura, D. W. McCamant, S. Yoon, D. B. Wandschneider and R. A. Mathies, Science, 2005, 310, 1006-1009.

3 D. Polli, P. Altoè, O. Weingart, K. M. Spillane, C. Manzoni, D. Brida, G. Tomasello, G. Orlandi, P. Kukura, R. A. Mathies, M. Garavelli and G. Cerullo, Nature, 2010, 467, 440. 
Stefan Haacke replied: (1) For rhodopsin, the group of Richard Mathies (UC Berkeley) in collaboration with M. Olivucci and I. Schapiro, have very recently shown ${ }^{1}$ that the phase of the HOOP of the hydrogens attached to C11 and C12 is critical to the isomerisation quantum yield. This was demonstrated by a decrease in the quantum yield when one of the hydrogens was deuterated, leading to a phase shift and thus to an unfavourable arrival time at the conical intersection. I believe that this experimental evidence, clearly supported by QM/MM modelling, establishes the HOOP mode to be the driving mode that provides the swing to the torsional motion.

(2) Such a time-dependent frequency analysis, for example using a sliding window FFT, is difficult to perform on the present data since the low-frequency vibrational modes are critically damped and we do not observe any more than 2 or 3 periods of oscillations. I would therefore refrain from carrying out such an analysis on the present data of ASR.

1 C. Schnedermann, X. Yang, M. Liebel, K. M. Spillane, J. Lugtenburg, I. Fernández, A. Valentini, I. Schapiro, M. Olivucci, P. Kukura and R. A. Mathies, Nat. Chem., 2018, DOI: 10.1038/s41557-018-0014-y.

Roberto Improta asked: How do you explain the effect of the point mutations you discussed on the excited state lifetime?

Stefan Haacke responded: As I indicated in the talk, two possible explanations can be invoked, but neither can explain the trends we observe in particular for the dramatic shortening of the excited state lifetime in L83Q. The response of the protein environment to the charge translocation on the PSBR builds up a large excited state difference dipole moment, with the positive end pointing towards the ionone ring. We know from the blue-shift of the S0-S1 transition in L83Q that the negative end of the $\mathrm{Q}$ dipole points towards the Schiff base, and thus rather opposes a repulsive interaction on the excited state, and even more so on the torsional motion, because the latter is predicted to increase the difference dipole moment. ${ }^{1}$

Another explanation relates to the mixing of S1 and S2, which, in the case of them being degenerate, was predicted to slow down the isomerisation. ${ }^{2,3}$ According to those studies, when we extrapolate their findings to AT PSBR in solution, the stabilisation of the SB positive charge due to Q83 would lead to a slowing down of the reaction rather than to the shortened excited state lifetimes that we have observed. Therefore, our understanding is clearly incomplete at this point.

1 F. Melaccio, N. Calimet, I. Schapiro, A. Valentini, M. Cecchini and M. Olivucci, J. Phys. Chem. Lett., 2016, 7, 2563-2567.

2 B. Demoulin, S. F. Altavilla, I. Rivalta and M. Garavelli, J. Phys. Chem. Lett., 2017, 8, 44074412.

3 M. Manathunga, X. Yang, Y. Orozco-Gonzalez and M. Olivucci, J. Phys. Chem. Lett., 2017, 8, 5222-5227.

Tolga Karsili remarked: Upon point mutation, the modifications of the gradients (and thus the topographies) of the potential energy surfaces associated with trans-cis isomerization may also lead to modifications in the local topography around the conical intersections associated with such an isomerization. As a result, do the authors expect point mutation to have an effect on the branching into the cis and trans ground state minima following internal conversion at these conical intersections? 
Stefan Haacke replied: Yes, we do expect changes, and it would be very interesting to look into this. The method that we used to determine the isomerization quantum yield in the wt, as explained by A. Cheminal et al., ${ }^{1}$ will be applied to the mutants as well. It should be noted that we have found remarkably low quantum yields of below $20 \%$ for the wt. More interestingly, it was found that the slower $\mathrm{AT} \rightarrow 13 \mathrm{C}$ isomerization has a higher quantum yield than the reverse process. This is required in order to make light-adaptation happen, as is needed for the biological function of the protein, which is a light-intensity sensor that controls lightlevel dependent protein expression.

1 A. Cheminal, J. Léonard, S-Y. Kim, K-H. Jung, H. Kandori and S. Haacke, Phys. Chem. Chem. Phys., 2015, 17, 25429-25439.

Rienk van Grondelle queried: Why is the role of vibrations ignored in the explanations that you have shown? I could imagine some vibrations in the few hundred per $\mathrm{cm}$ range coupled to the isomerization.

Stefan Haacke answered: I agree that these low-frequency vibrations are related to the isomerization reaction, and we can even show in the case of the most rapid reaction that they are launched by the latter. Therefore, they are a consequence of the ultrafast speed of the reaction which, in turn, for reasons that we don't quite understand for now, is enabled by specific mutations, in particular for L83Q.

Igor Schapiro noted: The most blue shifting mutant that you have found for Anabaena sensory rhodopsin is L83Q. This corresponds to the L105Q mutant in a related retinal protein that we have studied: proteorhodopsin. In proteorhodopsin there are two variants that have different amino acids at position 105 because the two variants are present at different depths of the ocean. Therefore, it seems like nature has selected the same mutant to adapt the bacteria in the ocean.

It is interesting that you have found an effect of this mutant on the excited state lifetime. I'm wondering, have you looked at the effect of the long lived intermediates? Is there any effect in this mutant on proton pumping?

Stefan Haacke responded: This is indeed an interesting question, and I thank you for pointing out the analogy with proteorhodopsin.

We have not studied the long-lived intermediates thus far. I will ask my partners (Prof. K. H. Jung and H. Kandori) whether they have any information on these for L83Q.

Ravindra Venkatramani opened the discussion of the paper by Ganga Periyasamy: I have two questions regarding your calculations of charge transport in peptidomimetics.

(1) Have you considered the influence of peptide conformational change/ dynamics on their charge transport properties?

(2) In your calculations of the hole transfer kinetics you neglect reorganization energy effects and justify this by arguing that the peptide geometry does not change significantly with the charge state. However, the dominant 
contribution to reorganization energy should come from the outer-sphere component which includes the solvent and this cannot be neglected. Can you comment on how you could include the outer sphere reorganization energy effects and how this might influence the trends in the charge transport kinetics for your peptidomimetics?

Ganga Periyasamy replied: (1) Yes, we have indeed carried out a conformational search for the neutral and cation geometries and have considered the lowest energy conformer for further studies into hole migration dynamics.

(2) The reorganization energy has been calculated in both gas and PCM media and was found to vary negligibly. Furthermore, the computed structural parameters show an insignificant structural change between the neutral and optimized cation geometries.

We agree that the dominant contribution of reorganization comes from the outer-sphere component, which has not been considered in the dynamics study. The outer-sphere mechanism due to the solvation environment in the hole migration dynamics might alter the timescale, which is not included. Moreover, we have discussed only the relative timescale with respect to unsubstituted ureido peptides and have not commented on the absolute timescale.

P. I. Pradeepkumar asked: What would the effect of the secondary structures (helix/sheet) of proteins be on the charge transfer process?

Ganga Periyasamy answered: The structure decides the hole migration timescale and the presence of weak interactions in the helix and sheet could increase the time scale for hole migration, as we have observed for one of the bent peptide structures in our previous work. ${ }^{1}$

1 S. Joy, V. V. Sureshbabu and G. Periyasamy, J. Phys. Chem. B, 2016, 120, 6469-6478.

Ankona Datta enquired: Could you provide any chemical insights into why hole migration is faster in the halogen substituted chromophores compared with those substituted with nitro groups? Do you observe any trends in hole migration rates for the halogen substituted chromophores?

Ganga Periyasamy responded: The change in electronegativity is inversely proportional to the hole migration time scale, where halogens are highly electronegative compared to $\mathrm{NO}_{2}$ and benzene substituents. This is clearly reflected in our time scale.

Rienk van Grondelle asked: What is the physical basis that determines this extremely fast timescale?

Ganga Periyasamy replied: The appropriate orbital overlap between donor and acceptor units is the reason for the faster timescale. This is made apparent by the correlation between the timescale and functional groups.

Javier Segarra-Martí remarked: In the paper you report values for the first ionisation potentials of the different systems; did you check the higher-energy 
doublet states as well? If they are close in energy, they can modulate the coherence formed upon absorption.

Ganga Periyasamy answered: We have computed the higher energy doublet states and found a larger energy difference between the two states. Hence we have considered only one state in our hole migration dynamics.

John Helliwell communicated: With regards to the anticipated usage of the authors' chosen peptide in molecular electronics, I estimate that the speed of an electron in their peptide is $\sim 2 \times 10^{5} \mathrm{~m} \mathrm{~s}^{-1}$. By contrast, the electron speed in graphene has been measured by Chuang et al. ${ }^{1}$ who observed an electron velocity at the Dirac point of $1.093 \times 10^{6} \mathrm{~m} \mathrm{~s}^{-1}$, which is the fastest velocity recorded for all known carbon materials. Therefore, what advantages does your peptide have for molecular electronics applications?

1 K-C. Chuang, R. S. Deacon, R. J. Nicholas, K. S. Novoselov and A. K. Geim, Philos. Trans. R. Soc., A, 2008, 366, 237-243.

Ganga Periyasamy communicated in reply: Numerous candidates in different areas have been investigated in order to improve them for their use in molecular electronics. Graphene and peptides are used in two different areas. However they are interlinked, as peptides have been used as functionalizing units for graphene. ${ }^{1}$ Our work highlights the preliminary studies into how charge transfer, through various functionalizing units containing peptido-mimetics, can be triggered by an electrochemical and optical pulse, which might be anchored to any metal or graphene surface. Charge transfer in peptidomimetics occurs through the backbone and the peptidomimetics used in this work have the advantage of being directional. Furthermore, the timescale of peptides can be appropriately controlled using functional units and conformers.

1 J. Katoch, S. N. Kim, Z. Kuang, B. L. Farmer, R. R. Naik, S. A. Tatulian and M. Ishigami, Nano Lett., 2012, 12, 2342-2346.

Priyadarshi Roy Chowdhury communicated: How is the substituent effect on the timescale of hole migration evaluated?

Ganga Periyasamy communicated in reply: The substituent changes the wave function (orbital) patterns, which have been used for following the evolution of the created hole. As a result the timescale of hole migration changes according to the nature of the functional groups at the donor and acceptor ends.

Rienk van Grondelle opened a general discussion of Stefan Haacke, Ganga Periyasamy, Arijit De and Gebhard F. X. Schertler's papers: Can the speakers first tell us what they believe to be the general theme of their presentations? What was the strongest example of a biologically relevant photophysical reaction?

Stefan Haacke responded: In my opinion, a common denominator was the quest to highlight and understand the influence of the protein environment on the photo-reaction. 
Ganga Periyasamy replied: In this session, we have discussed photo physical processes on the ultrafast timescale.

Charge and energy transfer with fast timescales are important and can be affected by various parameters such as the structure, solvent and counter ion. To study these properties, a wide range of experimental and computational methodologies have been used and integrated appropriately. The studies have helped to address many of these faster timescale processes.

There are many examples of photophysical reactions in nature. In my opinion the strongest example is the 'photosystem', where the charge transfer and energy transfer dynamics occur in a controlled and selective manner. Photosystems contain a unique protein, with nature choosing the chlorophyll structure and dynamics according to its environment in order to obtain the most efficient charge transfer dynamics.

Arijit De answered: All four of us talked about the dynamics of chromophores inside proteins upon light absorption and how the local protein environment fine-tunes these dynamics. I think the strongest example of a biologically relevant photophysical reaction is photosynthetic light harvesting.

Rienk van Grondelle asked Stefan Haacke, Arijit De and Gebhard F. X. Schertler: How does the environment dynamically control the speed of a reaction? In your papers you observe very different speeds and coherences. There are big differences between the different systems; could this be due to different dynamic control by the environment?

Stefan Haacke answered: As I have discussed in my answer to a previous question, there are two explanations for how the protein environment may modify the excited state potential energy surface (PES) in such a way that the excited state lifetime is shortened. The basic picture we have in mind is that a larger negative slope of the PES will better accelerate the wavepacket, thus removing it faster from the FC zone and promoting it faster along the reaction coordinate towards the intersection with the photoproduct ground state. However, on the basis of the standard models of retinal-chromophore interactions (e.g. dipole-charge), an opposite trend would be expected, i.e. a longer excited state lifetime for L83Q and the other mutants.

One may now speculate about steric effects, like the mutations leading to a pretwist of the AT retinal in the ground state, which would accelerate the reaction. Clearly, we need to obtain more structural information about the mutants.

Arijit De replied: It certainly depends on the (dynamic) coupling of the chromophores to their (immediate) environment, but I have a different view on the control of the speed of energy transfer. I think there is no simple answer to it as we simply do not know what biological systems have been trying to optimize over the course of evolution. It may be the case that photosynthetic organisms have optimized something else (e.g. fast adaptation to changing sunlight flux, which is more crucial for their survival) by tuning the local environment around the chromophores (pigments), and so the difference in the speed of energy transfer is just a by-product of this process. In other words, the environment evolved in such a way not to control the speed of energy transfer but to control something else. 
Gebhard F. X. Schertler replied: For retinal proteins we are only just beginning to understand how retinal isomerization is coupled to the earliest changes in the protein. It is clear that the protein environment is the source of the increased quantum yield and stereoselectivity of the retinal isomerization. From analysing the Raman spectroscopy results, it seems very likely that particular vibrational modes of the chromophore are essential for both effects. We also have observed using dynamic X-ray scattering and time resolved crystallography that correlated motions are induced very early in the protein. It is, however, unclear whether these are either directly coupled to the first conformational changes or are a mechanism for dissipating the surplus energy from the absorbed photon to prevent damage.

Mahesh Hariharan returned to the discussion of the paper by Gebhard F. X. Schertler: You showed a couple of proteins where you had isolated and clustered water molecules within the protein pockets. Do you see any structural generalisation in the protein pocket that allows for single water molecules $v s$. clusters of water molecules?

Gebhard F. X. Schertler responded: There are a number of bioinformatics programs which are already used in drug discovery that predict water molecules and their energy states. ${ }^{1}$ These predictions have already been utilized in drug discovery. At this point I have not yet included these bioinformatic approaches but I have instead only relied on experimental observations of the X-ray densities.

1 A. Bortolato, B. G. Tehan, R. T. Smith and J. S. Mason, Methods Mol. Biol., 2018, 1705, 207232.

Richard Cogdell remarked: I would like to ask about water clusters as well. We have been looking at the high resolution structures of proteins; on the surfaces we sometimes find pentamers of water that interact/propagate. When you have looked at similar structures have you seen any correlation between what amino acids you observe and whether or not you observe pentamers?

Gebhard F. X. Schertler replied: We have not specifically looked at the arrangement of water molecules on the surfaces of proteins. Our study focuses on the highly occupied, well-ordered molecules inside the protein that have clear electron densities, as shown in the figures. There are always water molecules on the surfaces of the proteins, and some get added towards the end of the refinement of the X-ray structure. Their significance is hard to judge as they are very dependent on the refinement strategy, but I certainly believe that more specific water clusters on the surfaces of proteins exist.

Anthony Watts addressed Gebhard F. X. Schertler and Stefan Haacke: How does the dielectric constant affect each of your systems, and how can it be accommodated in each system?

Gebhard F. X. Schertler answered: This is an excellent question that is not addressable by structural biology at the moment, and which can only be answered using simulations and calculations, which are now more enabled by fast electron laser measurements. Dielectric constants are to some extent bulk properties 
which are hard to define for a single protein molecule. It is even more difficult for membrane proteins, where we have to consider the local dielectric constants of polar groups embedded in the lipid bilayer and the hydrophobic membrane protein itself.

Stefan Haacke replied: The dielectric constant is a central quantity for the photoisomerization of the PSBR (Protonated Schiff Base of Retinal) since the first action of light absorption is a large charge translocation leading to a dipole moment change of approximately $20 \mathrm{D}$. However, the relevant dielectric constant to be considered is not a global one, but that of the amino acids forming the retinal binding pocket. We have to think here of a dielectric constant that is heterogeneous since it varies strongly on an atomic scale, reflecting the hydrophobic and hydrophilic character of the side chains, and of course the nearby $\mathrm{H}_{2} \mathrm{O}$ molecules. It should be noted that the binding pocket is hydrophilic close to the Schiff base and hydrophobic at the opposite ionone ring end of the PSBR. This can be calculated or mapped, but what is really important is the high frequency limit, in the $10 \mathrm{THz}$ range, since this is the relevant timescale for the retinal binding pocket being polarized by the charge translocation of the PSBR. I'm not sure that we have any precise information about this quantity. We showed, in collaboration with Majed Chergui (EPFL) in the mid-2000s for bacteriorhodopsin, that the PSBR charge translocation induces an intra-protein Stark effect that can be measured by monitoring the absorption shift of W86. ${ }^{1,2}$ Interestingly, the effect persists over timescales of $>10 \mathrm{ps}$, i.e. after isomerization, thus indicating a long-lived change of the dielectric constant, which is possibly associated with the rupturing of the $\mathrm{H}$-bonding network or the dislocation of water molecules close to the moving Schiff base end.

1 S. Schenkl, F. van Mourik, G. van der Zwan, S. Haacke and M. Chergui, Science, 2005, 309, 917-920.

2 J. Léonard, E. Portuondo-Campa, A. Cannizzo, F. van Mourik, G. van der Zwan, J. Tittor, S. Haacke and M. Chergui, Proc. Natl. Acad. Sci. U. S. A., 2009, 106, 7718-7723.

Amitabha Chattopadhyay addressed Arijit De, Gebhard F. X. Schertler and Anthony Watts: I would like to extend Anthony Watts's question a bit more. He is not only talking about a dielectric medium, he is talking about a dielectric gradient across the membrane leaflet experienced by a membrane protein, which is a hallmark of biomembrane organization. In the long run, we need to incorporate this parameter into the system.

Arijit De responded: That is a very important point, thank you. I would like to make a comment that the dielectric constant is a bulk property whereas the local electrostatic contribution from the nearby residues on the chromophore is a nano-scale phenomenon. Therefore, the spatial variation of a local electric field, rather than the dielectric constant, is perhaps a more relevant quantity to look at.

Gebhard F. X. Schertler answered: I agree.

Anthony Watts replied: That is correct and thank you for this comment. Some attempts have been made at incorporating the dielectric gradient across and within membranes, but it is incredibly complex; it is not a smooth linear gradient 
anywhere (protein and membrane landscapes are highly contorted), and it can vary over very short ranges from 2 to 80 . Furthermore, such changes are not permanent and vary temporally, on timescales that are wide ranging (fs-ms, or slower). Some of these changes will indeed be coupled to photoinduced conformational changes in receptors, which is the topic of the meeting.

Rienk van Grondelle asked Anthony Watts: We know that there are other more efficient proton pumps out there. Why do you think the efficiency is so different between these fairly homologous proteins?

Anthony Watts answered: My guess is that there are differences in the energetics of charge separation in different systems. There is not much else that can be different. The differences are as a result of the positions and number of water molecules in the proton channel, and the residues inducing the charge separation that leads to $\mathrm{H}^{+}$and $\mathrm{OH}^{-}$production following retinal isomerization. The data that Gebhard F. X. Schertler presented seems to support such a notion.

Rienk van Grondelle returned to the discussion of the paper by Ganga Periyasamy: If this energy landscape is so determined by the interaction with water, then water will play a role in the dynamics. If you were to add water to your model, what do you think would happen?

Ganga Periyasamy answered: The determination of the energy landscape with water can be considered in two ways, either implicitly or explicitly considering water molecules. Implicit solvation might only alter the timescale, while explicit solvation could alter the mechanism, depending upon its position at the donor/ acceptor end. The explicit presence of water molecules in our structure requires a detailed study starting from a conformational search, which we are currently working on.

Anthony Watts addressed Stefan Haacke and Ganga Periyasamy: How does your work inform our understanding of photocurrent generation by photoreceptors? Very close analogues can generate very different photocurrents.

Stefan Haacke replied: Perhaps my understanding is wrong, but in my mind we can treat the photoisomerization sub-ps dynamics as being disconnected from the slower micro- and milli-second processes of proton translocation. The time scales are so different that they can be treated as independent. However, the quantum yield of the initial photoisomerization of course modulates the amount of photocurrent for a given incident power.

Ganga Periyasamy responded: The dynamics after excitation might be similar to the hole migration dynamics. However it purely depends on the physical and chemical nature of the photoreceptor. The generation of photocurrent in photoreceptors is influenced by many factors. A minimal change in the stoichiometry or substituent could trigger an inner and outer sphere structure reorganization mechanism. Similar to peptides, I expect that a conformational change will alter the timescale and the direction of photocurrent. 
Ankona Datta opened the discussion of the paper by Rajaram Swaminathan: The changes of the Prochar spectra with $\mathrm{pH}$ would be due to the protonation/ deprotonation of amino acids. In order to study aggregates you are changing the $\mathrm{pH}$ of the sample. The changes observed in the spectra could therefore be due to protonation/deprotonation, rather than aggregation. Can you clarify this point?

Do you see any shifts in your spectra with aggregation or $\mathrm{pH}$ changes?

Rajaram Swaminathan answered: HEWL aggregation was monitored with respect to time at a constant $\mathrm{pH}$. Therefore, changes in the ProCharTS due to protonation/deprotonation were ruled out while observing the aggregation kinetics at constant $\mathrm{pH}$. There was no shift in the ProCharTs spectra during HEWL aggregation in the wavelength range of $325-800 \mathrm{~nm}$. There was a shift in the spectra under alkaline conditions, due to the presence of tyrosine, in the wavelength range of $250-325 \mathrm{~nm}$. There were no marked shifts in the ProCharTS spectra with $\mathrm{pH}$.

Dimitra Markovitsi remarked: We have shown that the irradiation of DNA duplexes in the UVA spectral domain, where they exhibit very weak absorption related to charge transfer transitions, induces photochemical reactions. ${ }^{1,2}$ Do you expect that, analogous to DNA, peptides may also be damaged when irradiated at the red edge of their absorption spectra? If so, could this property be promising for therapeutical reasons, such as amyloid destruction?

1 S. Mouret, C. Philippe, J. Gracia-Chantegrel, A. Banyasz, S. Karpati, D. Markovitsi and T. Douki, Org. Biomol. Chem., 2010, 8, 1706-1711.

2 A. Banyasz, I. Vayá, P. Changenet-Barret, T. Gustavsson, T. Douki and D. Markovitsi, J. Am. Chem.-Soc., 2011, 133, 5163-5165.

Rajaram Swaminathan replied: This is an interesting thought. It is definitely worthwhile to explore photoinduced damage in proteins. We look forward to pursuing these experiments to obtain the answers.

Bern Kohler noted: It can be difficult to distinguish weak absorption by a solute from light scattering by aggregates. What evidence do you have that scattering can be neglected, and did you check that the weak absorbance measured at long wavelengths varies linearly with protein concentration?

Rajaram Swaminathan responded: The poor overlap of the observed spectra with the simulated Rayleigh scattering profiles (Fig. S6 in the ESI $\dagger$ ) provides the evidence that the observed spectra are not due to scattering. In order to avoid scattering from aggregates, the samples were centrifuged at $12000 \mathrm{~g}$ for $10 \mathrm{~min}$ and the supernatant was used to record the absorption spectra.

With regard to your second question, Fig. S7 in the ESI $\dagger$ shows that the weak absorption measured at long wavelengths (400-700 nm) increases linearly with protein concentration.

Rienk van Grondelle commented: With regard to the proteins that at high concentrations develop these 'charge transfer' bands, what happens to the fluorescence of these proteins under these conditions? 
Rajaram Swaminathan answered: The charge transfer bands are emissive. Work relating to the fluorescence features of these protein charge transfer bands is in progress.

Richard Cogdell remarked: Unfolded regions are key for many proteins. Do you think that the small size of the signals you are measuring means that you will be able to use them as a probe for unfolded regions?

Rajaram Swaminathan replied: Yes indeed. This is something that we wish to do. In order to amplify the low absorbance, we intend to increase the pathlength of the observation volume.

Ashley Hughes commented: One could use a $10 \mathrm{~cm}$ pathlength, low volume cell here.

Ilme Schlichting asked: What happens in $\mathrm{D}_{2} \mathrm{O}$ ?

Rajaram Swaminathan replied: We have compared the absorption of lysine solution in both pure water and $\mathrm{D}_{2} \mathrm{O}$. There is no change observed in the ProCharTS absorption intensity in $\mathrm{D}_{2} \mathrm{O}$ in comparison with that in water even after $24 \mathrm{~h}$ of incubation at room temperature. ${ }^{1}$

1 S. Prasad, PhD thesis, IIT Guwahati, 2017.

Himani Medhi communicated: What is nickel affinity gel (H0537) and what is the role of this material when used in the ProCharTS absorption spectra?

Rajaram Swaminathan communicated in reply: Nickel affinity gel is an immobilized metal-ion affinity chromatography product consisting of a proprietary quadridentate chelate on highly cross-linked beaded agarose charged with nickel (source: https://www.sigmaaldrich.com/). This gel was used to purify the hexa-histidine tagged proteins by affinity chromatography. There is no role of this gel in the ProCharTS absorption spectra.

Mahil L communicated: In aggregated protein is it possible to observe absorption due to charge transfer and due to aromatic amino acids separately? Can you please comment on the intensity of the absorption spectrum due to both charge transfer and due to aromatic amino acids? Why does the aggregated protein exhibit a higher intensity in the absorption spectrum compared to that of the normal one?

Rajaram Swaminathan communicated in response: Yes, we can separately observe the absorption due to charge transfer in aggregated proteins in the wavelength range of $325-800 \mathrm{~nm}$. However, it is not possible to separately observe the absorption arising from aromatic amino acids in the wavelength range of 250-325 $\mathrm{nm}$ in the charge transfer spectra. This is due to a significant overlap of the ProCharTs spectrum with the aromatic amino acid absorption spectra in this wavelength region. The intensity of the absorption spectrum (325-800 $\mathrm{nm}$ ) due to charge transfer is quite low in comparison with that of the 
aromatic amino acids (250-325 $\mathrm{nm})$. This is because aromatic chromophores possess much higher molar extinction coefficients in comparison with ProCharTS. The aggregation of protein creates new intermolecular contacts between the charged side chains of amino acids. This increase in the population of charged side chains that are in close proximity increases the ProCharTS absorption intensity in the aggregate.

Priyadarshi Roy Chowdhury communicated: What challenges do you face during an investigation of the structural aspects using protein charge transfer spectra?

Rajaram Swaminathan communicated in reply: Our work has shown that the changes in the protein charge transfer spectra correlate well with the changes observed in the circular dichroism data. However, at the present moment we are unable to derive specific structural signatures of the protein from the ProcharTS data. One challenge is to predict a low resolution 3D structure of a protein from its ProCharTS spectrum. Work in this direction is under progress in our lab.

Arijit De opened the discussion of the paper by Ravindra Venkatramani: As you have just mentioned, (intermolecular) solvation is important for estimating electron transfer rates, which you have not considered thus far (as this is a gas phase calculation). However, even in the absence of solvent, large (intramolecular) conformational change can also influence the rates of electron transfer, somewhat like I proposed as a model in my talk (DOI: 10.1039/ c7fd00187h), by driving one locally excited (LE) state to one (twisted) intramolecular charge transfer ((T)ICT) state. Have you considered any such effect in your (gas phase) calculations?

Ravindra Venkatramani responded: While we have not considered solvation in the present manuscript, we have carried out a detailed analysis of the effect of the protein and solvent environment on the absorption spectra of Lys/Glu amino acids in our previous study. ${ }^{1}$ Here, our calculations focus on the photoinduced direct CT transitions in the absorption profile of amino acids with charged sidechains. We have not investigated the subsequent excited state dynamics or relaxation pathways of the electrons in our systems.

1 S. Prasad, I. Mandal, S. Singh, A. Paul, B. Mandal, R. Venkatramani and R. Swaminathan, Chem. Sci., 2017, 8, 5416-5433.

Manas Sarangi remarked: This sort of charge transfer phenomenon has been very well reported elsewhere in the literature. What makes it special for these charge transport states to absorb over such a large range? How fast is this charge transfer?

Ravindra Venkatramani replied: While CT transitions in metal-ligand complexes and protein backbones have been examined previously, we and our collaborators are the first to report photoinduced CT processes involving charged amino acids. ${ }^{1}$ In fact, our present study examines the absorption spectral range for CT transitions in charged amino acid monomers and attributes it to the 
backbone conformational flexibility of chromophores (Fig. 7). On the other hand, our previous study ${ }^{1}$ showed that the interactions between charged amino acid sidechains in a protein can further significantly extend the absorption range of the CT transitions to visible wavelengths. The charge separations we examine are photoinduced vertical transitions, which are virtually instantaneous (on the order of femtoseconds) relative to any nuclear motion.

1 S. Prasad, I. Mandal, S. Singh, A. Paul, B. Mandal, R. Venkatramani and R. Swaminathan, Chem. Sci., 2017, 8, 5416-5433.

Carlos E. Crespo-Hernández asked: Have you investigated how the level of theory used (i.e. other density functionals that can be used to describe charge transfer states such as LC- $\omega$ PBEh or wave-based versus density-based theories) may affect the excitation energies calculated in this study?

Ravindra Venkatramani answered: We have compared the computed TDDFT/ CAM-B3LYP/6-31++G(d) spectra of charged amino acid monomers (Lys cation/ Glu anion) with those computed with two other range-corrected hybrid functionals (LC- $\omega$ PBE or wB97XD) for multiple amino acid conformations. For Lys, the CAM-B3LYP excitation energies are on average $\sim 1.6 \mathrm{eV}$ lower than those obtained from LC- $\omega$ PBE and $\sim 0.5 \mathrm{eV}$ lower than those obtained from wB97XD. For Glu, the CAM-B3LYP excitation energies are on average $\sim 1.4 \mathrm{eV}$ lower than those obtained from LC-wPBE and $\sim 0.5 \mathrm{eV}$ lower than those obtained from wB97XD. The CT excitation energies obtained from all three range-corrected hybrids are much higher (on average $\sim 1.8-3.4 \mathrm{eV}$ higher for Lys and $\sim 2.2-$ $3.4 \mathrm{eV}$ higher for Glu) than those obtained from B3LYP. We have not yet compared the TDDFT results of our paper with high level wavefunction based approaches such as coupled cluster methods.

Mike Jones remarked: You briefly mentioned the fate of the electron and damage. Could the formation of these states actually have a beneficial physiological effect?

Ravindra Venkatramani responded: At present we do not have any computational or experimental data on the lifetime of the charge transfer excitons in our systems or on the probabilities of charge separation and recombination after photoexcitation. However, given that these transitions occur in charged amino acid rich proteins, we can speculate that an irreversible exchange of electrons between the peptide backbone and charged amino acid sidechains to create free charges could alter the surface electrostatics and sidechain interactions. The results of such photoexcitations could either be detrimental (e.g. disruption of DNA-protein interactions) or beneficial (e.g. hindering pathological aggregation of intrinsically disordered proteins), depending on the protein involved and the biological context. On the other hand, fast recombination of the electron-hole pair could efficiently quench the photoexcitations leading to no perceivable effect on either the protein structure or dynamics.

Joshy Joseph asked: Can you please comment on the fluorescence probability of these systems? 
Ravindra Venkatramani replied: Thus far, we have only examined the absorption spectra for charged amino acids and not the fate of the excited electrons and their relaxation pathways. Thus, we are unable to comment on the fluorescence in these systems at present.

Dimitra Markovitsi addressed Rajaram Swaminathan and Ravindra Venkatramani: Does excitation at the red edge of the peptide absorption spectrum give rise to fluorescence? In such a case, a time-resolved study, such as those performed for DNA with UVA excitation, ${ }^{1,2}$ could help better characterize the corresponding electronic transitions.

1 A. Banyasz, I. Vayá, P. Changenet-Barret, T. Gustavsson, T. Douki and D. Markovitsi, J. Am. Chem. Soc., 2011, 133, 5163-5165.

2 A. Banyasz, T. Gustavsson, D. Onidas, P. Changenet-Barret, D. Markovitsi and R. Improta, Chem.-Eur. J., 2013, 19, 3762-3774.

Rajaram Swaminathan responded: Our initial results show that ProCharTS emits fluorescence upon excitation at the red edge $(600 \mathrm{~nm})$. Time-resolved studies would indeed prove useful to better understand these transitions. This work is currently under active progress in our lab.

Ravindra Venkatramani responded: We have not yet computationally examined the relaxation pathways or dynamics of the excited electron in our charged amino acid spectra. However, experimentally exciting at the red-edge of the charged amino acid rich protein spectra to check for fluorescence would be very interesting. Thank you for your helpful suggestion for further characterizing the spectra and for your references to your previous studies on DNA UVA excitation which were relevant to this discussion.

Ravi Kumar Venkatraman commented: It is quite remarkable that one can sample the various conformational degrees of freedom of amino acids in native protein structures using classical-molecular dynamics simulations (c-MDS) and then measure their inhomogeneity in the charge-transfer (CT) absorption spectra using time-dependent density functional theory calculations. However, my question is regarding the computational methodology that was adopted in your paper. Generally one calculates the vertical excitation using the same level of theory for both the ground and excited electronic states. In this paper, the ground state structures were obtained from either c-MDS or X-ray crystal structures. Would you expect that this methodology will modify your results?

It is well known that many functionals fail to predict the CT energies owing to over-stabilization of the ground state structure. ${ }^{1}$ Therefore, predicting the ground state structure is critical. To mitigate this problem we have adopted a methodology in our recent paper, ${ }^{2}$ where instead of sampling intra-molecular conformational degrees of freedom we have sampled intermolecular (solute-solvent) conformational degrees of freedom. The low frequency conformational degrees of freedom sampled by c-MDS were frozen and a relaxed optimization along other high frequency vibrational modes was carried out using DFT calculations.

1 J. J. Eriksen, S. P. A. Sauer, K. V. Mikkelsen, O. Christiansen, H. J. A. Jensen and J. Kongsted, Mol. Phys., 2013, 111, 1235-1248.

2 V. R. Kumar, C. Verma and S. Umapathy, J. Chem. Phys., 2016, 144, 064302. 
Ravindra Venkatramani replied: Vertical excitations by definition involve the photoinduced promotion of electrons to excited states at the ground state nuclear geometry of molecules. Thus, the initial and final electronic states of the transitions for our monomer amino acid fragments are computed at a single level of theory (TDDFT/CAM-B3LYP/6-31++G(d)) in the absorption spectra calculations. To sample the ground state nuclear geometries for our monomer amino acid fragments, we use classical molecular dynamics simulations on entire solvated proteins and extract individual amino acid conformations from the simulated structures. This method samples monomer amino acid conformations within an explicit solvated protein environment which is the system of interest in our present study. We do not carry out any additional DFT optimizations on the extracted amino acid fragments, as such calculations will lead to unnatural amino acid conformations in the absence of the protein environment. Constrained DFT optimization strategies such as those employed by Kumar et al. ${ }^{1}$ also cannot account for the environment of the protein during geometry relaxation. We note that Kumar et al. ${ }^{1}$ have applied their DFT optimizations to a neutral molecule (benzophenone) in a homogenous solvent environment which can be mimicked with a mixed explicit + implicit solvation model. In contrast, our aim is to examine charged amino acid chromophores which interact with other charged and polar sidechains in the protein as well as with water. In this case, classical molecular dynamics simulations appear to be the best way to sample the ground state amino acid conformations.

With regards to the comment on the TDDFT methodology, we have employed the CAM-B3LYP functional which provides a reasonable description of CT transitions in organic molecules. A discussion of this and accompanying references can be found in the subsection "Critical discussion of modelling assumption and caveats" in the "Methods" section of the present manuscript. We do not perform geometry optimizations using DFT/TDDFT as carried out by Eriksen et al. ${ }^{2}$ which should reduce errors as the ground state geometries of our chromophores do not suffer from DFT limitations. Furthermore, in the present work we compare relative trends in the absorption profiles for a range of chromophores and not absolute energy gaps, which was the objective of Eriksen et $a l^{2}$

1. V. R. Kumar, C. Verma and S. Umapathy, J. Chem. Phys., 2016, 144, 064302.

2 J. J. Eriksen, S. P.A. Sauer, K. V. Mikkelsen, O. Christiansen, H. J. A. Jensen and J. Kongsted, Mol. Phys., 2013, 111, 1235-1248.

Anvy Kuriakose remarked: The interactions between lysine and lysine and lysine and glutamate were discussed in the paper, but glutamate-glutamate interactions were never mentioned. I wondered why these were not mentioned.

Ravindra Venkatramani answered: I presume you are referring to the systems considered in our previous study. ${ }^{1}$ In that study, Glu-Glu interactions have also been considered and discussed. Please see Fig. 5 (panel e), Fig. 6 (middle row b) and ESI Fig. S17† along with the associated discussion on pages 5426-5427 in that study.

1 S. Prasad, I. Mandal, S. Singh, A. Paul, B. Mandal, R. Venkatramani and R. Swaminathan, Chem. Sci., 2017, 8, 5416-5433.

Vishnu V communicated: Why do only lysine and glutamate show charge transfer? 
Ravindra Venkatramani communicated in reply: Lys and Glu possess charged sidechain groups $\left(\mathrm{NH}_{3}{ }^{+} / \mathrm{COO}^{-}\right)$which polarize the filled and empty orbitals across the chromophores (Fig. 3 of the present manuscript). Thus, during photoexcitation, electrons are spatially separated from the hole that they leave behind, giving the transitions a backbone-sidechain CT character. Our present study shows that such photoinduced CT transitions are not limited to only Lys/ Glu, but are intrinsic properties of all amino acids with charged sidechains. We list such chromophores in Fig. 1 of the present manuscript.

John Helliwell communicated: At physiological pH the authors assume a particular (charged) protonation state for Asp and Glu. For His they rightly consider the two possible protonation states. Due to the local structure environment the $\mathrm{p} K_{\mathrm{a}}$ values of Asp and Glu can vary from the values for the free amino acids. ${ }^{1}$ Therefore, in addition to His, both protonation states of Asp and Glu must be accounted for. Do the authors plan to extend their calculations to do that?

1 S. J. Fisher, J. Wilkinson, R. H. Henchman and J. R. Helliwell, Crystallogr. Rev., 2009, 15, 231-259.

Ravindra Venkatramani communicated in reply: I fully agree with Professor Helliwell that the $\mathrm{p} K_{\mathrm{a}}$ values of amino acids can deviate significantly within proteins from their free solution values and that Glu/Asp residues may be uncharged. We considered the effect of different charged states on the spectra of Lys and Glu in our previous study ${ }^{1}$ and showed that in their uncharged states the lowest energy transitions for these amino acids do not possess backbone-sidechain CT character. We anticipate the same trends to hold for the charged/uncharged states of all of the amino acids considered in the present manuscript including Asp. Thus, choosing the right protonation states for the amino acids sampled from protein folds is very important to quantitatively interpret the spectra arising from proteins rich in charged amino acids. We thank Professor Helliwell for bringing our attention to his paper, which was relevant for this discussion.

1 S. Prasad, I. Mandal, S. Singh, A. Paul, B. Mandal, R. Venkatramani and R. Swaminathan, Chem. Sci., 2017, 8, 5416-5433.

Tolga Karsili opened the discussion of the paper by Igor Schapiro: Upon comparing the relaxed CASSCF and CASPT2 potential energy profiles in Fig. 2, I noted that the CASPT2 profiles show an initial rise in potential energy followed by a subsequent decline. The inevitable barrier returned from this rise and decline in potential energy may indicate a change in or mixing of the electronic configuration(s). This same profile is however unobserved in the CASSCF relaxed potential energy profiles. Since the QM/MM surface-hopping simulations are based on onthe-fly CASSCF energies, are the authors confident that the obtained timescale for isomerization is not underestimated?

Igor Schapiro answered: There is indeed a small barrier in the case of the relaxed scan that was re-computed using CASPT2. However, the height of this barrier is significantly smaller than the excess energy that becomes available to the retinal chromophore upon relaxation from the Franck-Condon point. 
Therefore, I believe there will be no significant difference in the timescale of the isomerization.

Tolga Karsili asked: Is there a future plan to propagate more trajectories in order to obtain better statistics? If so, how will the initial conditions of such trajectories be defined?

Igor Schapiro answered: Yes, we plan to run more trajectories in the future to obtain statistically significant results. Up to now we have computed 10 trajectories (Fig. 2). The initial conditions were generated by computing a long classical trajectory which sampled the chromophore and the protein phase space. Then we took snapshots from this trajectory and used the geometries for the ground state $\mathrm{QM} / \mathrm{MM}$ trajectories. Those were subsequently used for the excited state QM/MM trajectories after a sampling period of half a picosecond.

John Helliwell communicated: How do the you assess the statistical significance of the structural changes along your calculated trajectories, such as in Fig. 5 in your paper?

Igor Schapiro communicated in reply: We are in the process of running more trajectories to address the statistical relevance of the so called " $0 \mathrm{~K}$ trajectory" that is presented in the manuscript. The sampling of the initial conditions and the

\section{QM/MM trajectories}

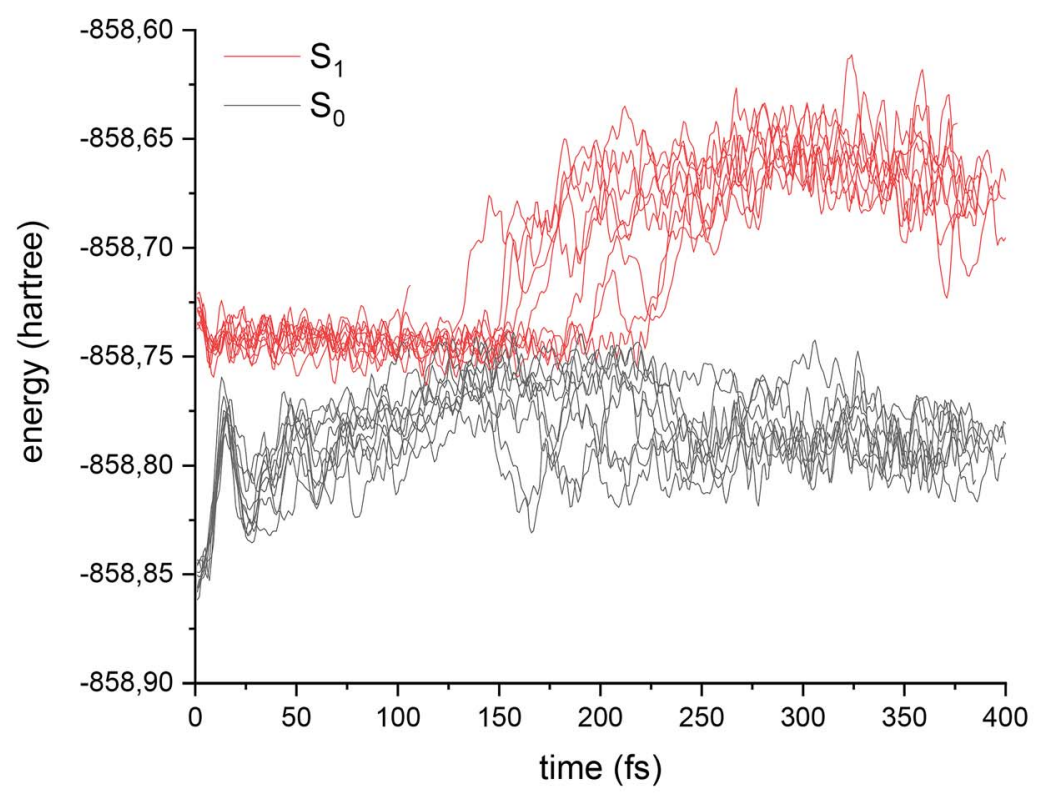

Fig. 210 QM/MM trajectories starting from different initial conditions. 
excited state dynamics are computationally demanding. At the moment we have computed 10 trajectories, which can be seen in Fig. 2 in my response to the previous question. These 10 trajectories show the same mechanism of isomerization but have varying excited state lifetimes.

Carlos E. Crespo-Hernández commented: Does the size of the QM region in your QM/MM calculations affect the excited state dynamics predicted for the green-absorbing proteorhodopsin? In other words, what is the minimum number of moieties (i.e. retinal chromophore, side chains and water molecules) that needs to be included in the QM region in order to properly describe the dynamics of green-absorbing proteorhodopsin when carrying out QM/MM calculations?

Igor Schapiro responded: The QM region in our setup contains the full retinal chromophore and a part of the lysine sidechain, which together account for more than 50 atoms. This is close to the limit of what we can do in excited state dynamics simulations. Hence, it is not possible to extend the QM region much further. We did however carry out some tests where we extended the QM region for the calculations of the excitation energies. In these single point calculations we did not observe any significant change if neighbouring side chains were included. Hence, we assume that the results will not be affected by a larger QM region.

Stefan Haacke queried: Is it correct to think of one reaction coordinate or should we not consider many low frequency modes and their energetics for promoting isomerization?

Igor Schapiro replied: One reaction coordinate is not enough to describe the photoisomerization. This reaction is triggered by a photon which is absorbed by the retinal. Consequently, the charge distribution of the retinal changes. This leads to a rearrangement of the nuclei and an inversion of the bond length alternation; double bonds become single bonds and single bonds become double bonds in the excited state. The next step is the onset of the rotation of two double bonds according to the bicycle pedal mechanism. Therefore we need to consider multiple reaction coordinates for the description of the isomerization.

Roberto Improta asked: Do you know if any dynamical studies of retinal exploiting TD-DFT exist? What is the picture that they provide with respect to the CASPT2 results?

Igor Schapiro answered: Yes, there are a few studies which use linear response TD-DFT to describe retinal in the excited state. However, this method cannot describe the degeneracy at the conical intersection or in its close proximity. Since the excited state trajectories evolve through such regions of the potential energy surface we have not used TD-DFT. However, there are some promising new methods that use the spin-flip approach or spin-restricted ensemble-DFT. Once they become available we will be happy to look into it.

Anthony Watts queried: Is there any other retinal binding protein that has a similar restricting residue to Tyr200 in your system? 
Igor Schapiro responded: I have not checked if this residue is conserved in other retinal proteins. We need to do a sequence alignment and compare it with proteorhodopsin.

Ilme Schlichting remarked: Tyrosine 120 in proteorhodopsin corresponds to tyrosine 185 in bacteriorhodopsin. They are both at a van der Waals distance from the retinal. Should we not see the same effect there?

Igor Schapiro replied: Thank you for pointing this out. We have not looked at bacteriorhodopsin in as much detail as for the green-absorbing proteorhodopsin. However, I would expect that the interaction between retinal and tyrosine 185 is also important for bacteriorhodopsin.

Anvy Kuriakose communicated: I believe you mentioned in your discussion that "You don't do any potential energy minimizations for molecular dynamics; you just run the simulation, lean back and wait for the results." I thought that energy minimization was the first step of molecular dynamics?

Igor Schapiro communicated in reply: You cited me correctly. However, this citation was part of a reply to the question of Stefan Haacke. He asked about the difference between the simulations I have presented and a minimum energy path. In this context I replied that a minimum energy path calculation is a static approach where specific points on the potential energy surface are optimized. In contrast, molecular dynamics simulations describe a propagation in time which does not necessarily describe a minimum energy path.

Furthermore, energy minimization is not the first step in a molecular dynamics simulation. The geometry that is chosen as a starting point for such a simulation can be obtained from an energy minimization, but it doesn't have to be.

Rienk van Grondelle opened a general discussion of the papers by Rajaram Swaminathan, Ravindra Venkatramani and Igor Schapiro, and addressed Igor Schapiro: I am confused about your dynamics. The fast oscillation does not seem to drive the isomerisation as this is much slower. What could drive the isomerisation? How big is the hydrogen displacement? Do you know from Raman analysis etc. which frequency is coupled to the optical transition? This could what drives isomerisation. If you look at the PES, there could be another parabola that drives isomerisation.

Igor Schapiro replied: This is a misunderstanding. The fast modulation of the potential energy is due to the bond stretching mode. We see this in the simulation because we calculate only one retinal chromophore at a time. In experiments you measure an ensemble, which means that this fast oscillation is averaged out or not resolved. What we know now is that the photon promotes retinal on the excited state where the stretching mode is activated. This is also called the inversion of the bond length alternation. Basically, the double bonds effectively become single bonds and vice versa. After that the $\mathrm{C} 13=\mathrm{C} 14$ bond is significantly stretched and we observe an onset of rotation around this double bond in our simulations. Therefore, my answer to your question is that the driving force of the 
isomerization is the combination of the stretching mode and the torsion of the double bond.

Bern Kohler returned to the discussion of the paper by Ravindra Venkatramani: It is striking that your predicted CT states lie more than $1 \mathrm{eV}$ below the HOMO-LUMO gaps calculated for your compounds. The much lower transition energies that you report for the anionic amino acid monomers make me wonder whether the omission of a solvent in your calculations could lead to spurious CT states. There are well-known pitfalls to predicting CT states using TDDFT calculations. ${ }^{1,2}$ What steps did you take to avoid these difficulties, and do the energies change significantly if you include a continuum solvation model?

1 A. Lange and J. M. Herbert, J. Chem. Theory Comput., 2007, 3, 1680-1690.

2 N. T. Maitra, J. Phys. Condens. Matter, 2017, 29, 423001.

Ravindra Venkatramani responded: While TDDFT with specific exchangecorrelation functionals may produce spurious low energy CT transitions, the deficiencies of these functionals in terms of their distance dependence that lead to such failures are well known and noted in both Neepa Maitra's review and John Herbert's study. We have used a range-separated hybrid functional (CAM-B3LYP) which provides a reasonable description of CT states in organic molecules and peptides. Furthermore, we verified that there are no spurious CT transitions in our calculated spectra by comparing computed excitation energies with the lower bound for CT excitation energies given in terms of the ground state energy gap and the exciton binding energy (see equations 4 and 5 in our paper). In general, as I mentioned in my response to Professor Crespo-Hernández's query, rangeseparated hybrid functionals produce much higher CT excitation energies than those obtained from B3LYP.

The issue of solvation in our systems is a bit involved. The objective of the present study is to compare the 'intrinsic' absorption features of charged amino acids as a function of their conformation within protein folds. In that sense, the absorption spectra calculated in the present manuscript are not directly experimental observables, but rather represent the essential electronic properties of charged amino acids which form the basis of the interpretation of the UV-Vis absorption of proteins that are rich in such residues. The experimentally observable spectra ${ }^{1}$ arise when many charged amino acids are tightly packed within a protein fold. Continuum solvation and QM/MM models are not applicable here, since they require a clear demarcation of the chromophore-solvent or QM/MM boundary which is not possible for these systems. The neighbourhood of each charged amino acid chromophore includes other charged protein sidechains and bound water, all of which can undergo changes in their charged states upon photoexcitation. Thus, the only way to assess the effect of the environment is to explicitly increase the size of the QM region and systematically sample the different charged states of the amino acids. We examined this issue in detail in our previous study ${ }^{1}$ and found that solvation can lead to two effects that can shift the wavelengths of CT transitions in opposite directions: (1) the screening/polarization effect which tends to localize CT donor/acceptor state wavefunctions and shift the transitions to higher energies, and (2) the electronic coupling effect which tends to delocalize CT donor/acceptor state wavefunctions and shift the $\mathrm{CT}$ transitions to lower energies. 
We would like to draw attention to the subsection "Critical discussion of modelling assumption and caveats" in the "Methods" section of the present manuscript, and our previous study ${ }^{1}$ which discusses the above considerations in more detail along with suitable references.

1 S. Prasad, I. Mandal, S. Singh, A. Paul, B. Mandal, R. Venkatramani and R. Swaminathan, Chem. Sci., 2017, 8, 5416-5433.

Richard Cogdell returned to the discussion of the paper by Rajaram Swaminathan: You can now easily synthesise the peptides that you design. If you were going to design some peptides, what would you design in order to give you a good testbed of how applicable your spectroscopy is going to be to real life peptides?

Rajaram Swaminathan replied: We could design peptides such that the amino (A) and carboxylate (C) groups of Lys and Glu, respectively, are positioned at chosen distances from each other. In this way we can establish a correlation between the distance between these charged groups and their ProCharTS absorbance.

Additionally, this approach can similarly also be repeated with AA and CC pairs. Furthermore, this can be extended to other charged residues such as His, Arg and Asp. Once all of these were designed, we would be in a better position to establish the spectral signatures in ProCharTS of the structural features that exist among the charged side chains in proteins.

Anthony Watts returned to the discussion of the paper by Ravindra Venkatramani: When you come to other residues, they too can have different $\mathrm{p} K_{\mathrm{a}}$ values. Do you consider those as well? Are the dielectric constants related?

Ravindra Venkatramani answered: We appreciate the fact that the $\mathrm{p} K_{\mathrm{a}}$ values of amino acids would be sensitive to the dielectric medium in which they are embedded. While the present manuscript deals with only the charged states of various amino acids, we have considered the non-charged states of Lys/Glu in our previous study. ${ }^{1}$ Our analysis shows that protonation of Glu or deprotonation of Lys amino acids eliminates the backbone-sidechain CT character of the lowest energy transitions. This is because the polarization of filled and empty orbitals across the chromophores (Fig. 3 in the present manuscript) is lost. A comparison of singly and doubly protonated His in the present study also confirms that the lowest energy excitations are backbone-sidechain CT transitions only for the latter.

1 S. Prasad, I. Mandal, S. Singh, A. Paul, B. Mandal, R. Venkatramani and R. Swaminathan, Chem. Sci., 2017, 8, 5416-5433.

\section{Conflicts of interest}

Gebhard F. X. Schertler is a co-founder of the leadXpro and InterAx biotechnology companies. There are no other conflicts to declare. 